\title{
Combine Simulation and Experiment EELS to Characterize Ionomer Conformation
}

Chen Wang ${ }^{1}$, Stephen J. Paddison ${ }^{2}$, and Gerd Duscher ${ }^{3}$

1. Division of Applied Research and Technology, National Institute for Occupational Safety and Health, Cincinnati, OH, 45226, USA

2. Department of Chemical and Biomolecular Engineering, University of Tennessee, Knoxville, TN 37996, USA

3. Department of Materials Science and Engineering, University of Tennessee, Knoxville, TN, 37996, USA

The development of modern electron energy-loss spectroscopy (EELS) techniques allows one to probe both local chemical compositional information and structural properties of polymeric materials [1]. High resolution EEL spectra acquired by a monochromatic TEM/STEM may be analyzed and compared with simulated spectra from first principle based calculations. The specific features in the low-loss and Kshell core-loss spectra of carbon are useful in characterizing the complex backbone conformations of perfluorosulfonic acid (PFSA) ionomers, such as Nafion ${ }^{\mathrm{TM}}$ and Aquivion ${ }^{\circledR}$ [2-3].

Chain conformations of PFSA ionomers were investigated with a monochromatic EELS on a $200 \mathrm{kV}$ Zeiss Libra 200 TEM/STEM. Membrane samples were prepared by cryo-microtome and examined in the cryo environment to minimize sample damage due to electron beam exposure. The EEL spectra were then fitted with Gaussian and Lorentzian functions using the Quantifit program [4].To better understand the structural and optical properties of these materials, density function theory (DFT) calculations were undertaken to compute energy-loss spectra in both low-loss and core-loss regions. The simulations were carried out with the GGA-PBEsol exchange-correlation functional. The polarization of the electromagnetic field was taken into account by varying the directions of the electric field vector of the incident electrons. The contributions from three directional components were then averaged to simulate the optical response of the polycrystalline systems.

Results using this approach to characterize the chain conformations of polytetrafluoroethylene (PTFE) and Nafion are shown in Figs. 1 and 2, respectively. The experimental spectra show several unique features in the low-loss region and the onset of the carbon $\mathrm{K}$ edge for PTFE. Similar features were observed for Nafion and further examined with DFT calculations. The spectral features including shape, width, and relative intensities of the characteristic peaks in the experimental spectra are well reproduced by the DFT calculations. By comparing the experimental and theoretical spectra, we have demonstrated an application of combining high resolution EELS and DFT calculations to discriminate changes of chain conformation and orientation in PFSA ionomers.

References:

[1] M.R. Libera and R.F. Egerton, Polymer Reviews, 50 (2010), 321.

[2] C. Wang, G. Duscher and S.J. Paddison, Microscopy, 63 (2014), 73-83.

[3] C. Wang, G. Duscher and S.J. Paddison, RSC Adv., 5 (2015), 2368-2373.

[4] G. Duscher, M.E. Hmielewski and J.D.O. Oduor, Microsc. Microanal., 15 (2009), 446-447. 


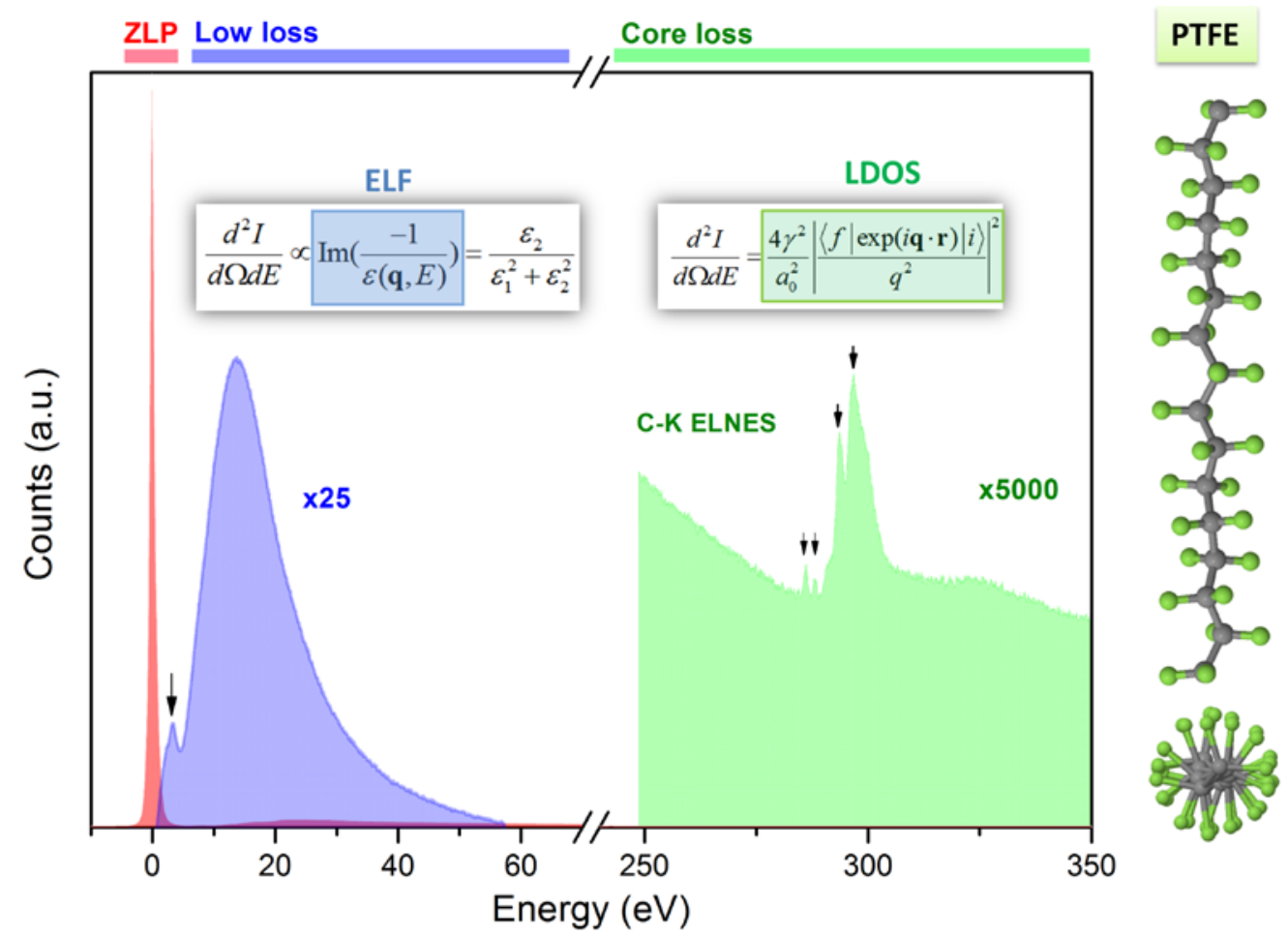

Figure 1. Experimental EEL spectra of PTFE show features in low-loss and near C-K edge. Energy loss functions (ELF) and local density of states (LDOS) of carbon were calculated to investigate spectral properties as a function of chain conformation.
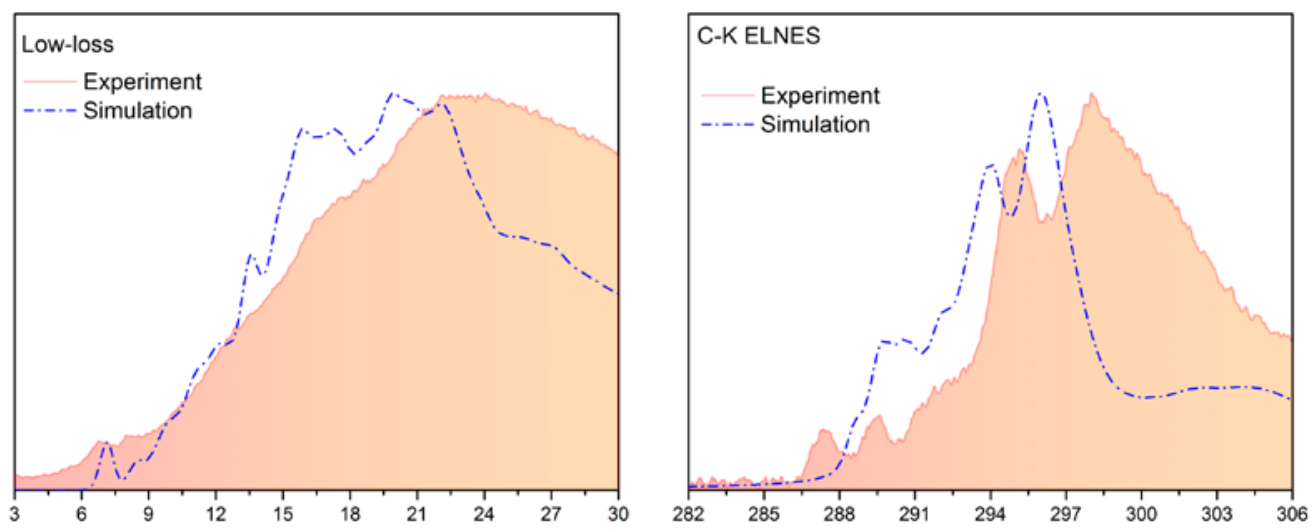

Nafion
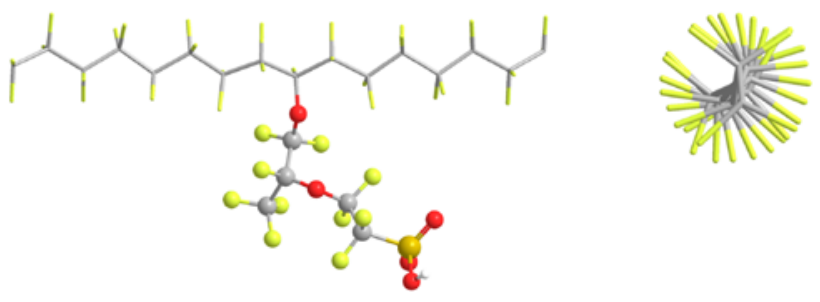

Figure 2. Comparison of low-loss and C-K ELNES from experiment and simulation for Nafion ${ }^{\mathrm{TM}}$. The gross features of experimental EELS can be reproduced from DFT calculations. 